\title{
Study of the Abundance Patterns in the Metal-Poor Stellar Stream
}

\author{
Hongjie $\mathrm{Li}^{1,2,3}$, Shuai Liang ${ }^{1,2}$, Wenyuan $\mathrm{Cui}^{1,2}$ and Bo Zhang ${ }^{1,2,4}$ \\ ${ }^{1}$ Department of Physics, Hebei Normal University, 113 Yuhua Dong Road, Shijiazhuang 050016, China \\ ${ }^{2}$ Hebei Advanced Thin Films Laboratory, Shijiazhuang 050016, China \\ ${ }^{3}$ School of Sciences, Hebei University of Science and Technology, Shijiazhuang 050018, China \\ ${ }^{4}$ Corresponding author. Email: zhangbo@ mail.hebtu.edu.cn
}

(Received February 18, 2012; Accepted September 12, 2012; Online Publication January 24, 2013)

\begin{abstract}
The chemical abundances of the metal-poor stars in the stellar stream provide important information for setting constraints on models of neutron-capture processes. The study of these stars could gives us a better understanding of the $r$-process nucleosynthesis and chemical composition of the early Galaxy. Using the updated main $r$-process and weak $r$-process patterns, we fit abundances in the stellar stream stars. The weak $r$-process component coefficients are almost constant for the sample stars, including $r$-rich stars, which means that both the weak $r$-process and Fe are produced as primary elements from Type II supernovae and their yields have nearly a constant mass fraction. The difference between the stream stars and $r$-rich stars is obvious. For the stream stars, the fact that the increased trend in the main $r$-process component coefficients as metallicity increases means a gradual increase in the production of main $r$-process elements relative to iron. This behaviour implies that the masses of progenitors for the main $r$-process are smaller than those of the weak $r$-process. Furthermore, we find that the metal-poor stream star HD 237846 is a weak $r$-process star.
\end{abstract}

Keywords: nuclear reactions, nucleosynthesis, abundances - stars: abundances

\section{INTRODUCTION}

The formation and evolution of the Galaxy have not been fully understood, although many studies have explored the problems for several decades. Early studies thought that our Galaxy formed through isolated collapse (Eggen, LyndenBell, \& Sandage 1962). The idea that the halo of the Galaxy may have formed partly by accreting some small fragments was proposed by Searle \& Zinn (1978). The stars related to these fragments could be recognisable as kinematic substructures and chemical composition among the Galactic halo stars. They should be concentrated into a number of coherent 'streams' in velocity space. Helmi et al. (1999) discovered a stellar steam in the solar neighborhood by detecting the distribution of angular momentum components. They estimated that about $10 \%$ metal-poor halo stars came from the stellar stream whose progenitor system was disrupted during the formation of the Galaxy. Because the abundance ratios of $[\alpha / \mathrm{Fe}]$ in the stream are similar to those of other halo stars, Kepley et al. (2007) suggested that the abundances of these stream stars are mostly enriched by Type II supernovae (SNe II). The stellar stream provides an excellent test bed for understanding the formation of our Galaxy. In this respect, a detailed study of elemental abundances, including the abundances of neutron-capture elements, in the stream is important.
The elements heavier than the iron-peak elements can be made through two neutron-capture processes: the $r$-process and the $s$-process (Burbidge et al. 1957). The $s$-process is further divided into two components, i.e., the main $s$ component and the weak $s$-component. The sites of the main $s$-component are thought to be in low- to intermediate-mass stars in the asymptotic giant branch (AGB; Busso, Gallino, $\&$ Wasserburg 1999). The most likely sites for the weak $s$ component are in core helium burning and shell carbon burning in massive stars. The $r$-process sites are associated with SNe II from massive stars, but it has not been fully confirmed (Cowan, Thielemann, \& Truran 1991; Sneden, Cowan, \& Gallino 2008). Observations of the very metal-poor halo stars CS 22892-052 (Sneden et al. 2003; Cowan et al. 2005) and CS 31082-001 (Hill et al. 2002; Honda et al. 2004) have revealed that their heavier neutron-capture elements $(Z \geq$ 56) are in remarkable agreement with the scaled solar system $r$-process pattern produced by the main $r$-process (Cowan et al. 1999; Truran et al. 2002; Wanajo \& Ishimaru 2006), but their lighter neutron-capture elements $(37 \leq Z \leq 47)$ seemed to be deficient (Sneden et al. 2000; Hill et al. 2002). This means that the $r$-process abundance pattern in solar system material cannot be explained by a single process. Based on the calculation of Galactic chemical evolution, Travaglio et al. (2004) concluded that another component from massive 
stars is needed to explain the solar abundance of Sr, Y, and $\mathrm{Zr}$. The corresponding process has the primary nature and is called the 'lighter element primary process (LEPP; see Travaglio et al. 2004 and Serminato et al. 2009) or 'weak $r$-process component' (Cowan et al. 2005; Ishimaru et al. 2005; Cowan \& Sneden 2006; Montes et al. 2007; Izutani, Umeda, \& Tominaga 2009). Zhang, Cui, \& Zhang (2010) analyzed the abundances of 14 metal-poor stars with metallicities $[\mathrm{Fe} / \mathrm{H}]<-2.0$ and found that the abundance patterns of both neutron-capture elements and light elements could be best explained by having a star form in a molecular cloud polluted by the weak $r$ - and main $r$-process material.

A quantitative understanding of the origins of $r$-process elements in the metal-poor stars is still difficult. In this respect, the stream stars are optimum sites for the study of the $r$-process because most stream stars have not been polluted by the $s$-process material. Recently, Roederer et al. (2010a) observed the abundances of 12 metal-poor stream stars. Their angular momentum components clumped together far more than the random distribution of halo stars, which was first reported by Helmi et al. (1999). All of the 12 members have angular momentum components with $500<L_{z}<1500 \mathrm{kpc}$ $\mathrm{km} \mathrm{s}^{-1}$ and $L_{\perp} \sim 2000 \mathrm{kpc} \mathrm{km} \mathrm{s}{ }^{-1}$, where $L_{z}$ is the angular momentum component of rotation for a given star's orbit and $L_{\perp}$ is the combination of the other two angular momentum components $\left(L_{\perp}=\sqrt{L_{x}^{2}+L_{y}^{2}}\right)$. Based on observations of metal-poor stream stars, Roederer et al. (2010a) concluded that the observed abundance of the heavy elements in most stream stars cannot be fitted well by the abundances from a single $r$-process, especially for the lighter neutroncapture elements $\mathrm{Sr}, \mathrm{Y}$, and $\mathrm{Zr}$. In order to better understand the origins of neutron-capture elements in the stellar stream, one must consider the contribution of the weak $r$-process. To make progress in our understanding of the neutron-capture processes, we study the element abundance patterns of the metal-poor stream stars, in which light elements, iron group elements, lighter and heavier neutron-capture elements are observed.

In this paper, we calculate the relative contributions from the individual neutron-capture process to the elemental abundances of nine stream stars in which elemental abundances of Eu have been observed. In Section 2, we obtain the two $r$ process components and extend the two abundance patterns to light elements. Using the updated weak $r$-component and main $r$-component, we calculate the relative contributions from the individual neutron-capture process to the elemental abundances in the stream stars. The calculated results are presented in Section 3. Our conclusions are given in Section 4.

\section{THE WEAK $r$-PROCESS AND MAIN $r$-PROCESS COMPONENTS}

Observational abundances in metal-poor stars show that sites producing the heavy $r$-nuclei do not produce iron group elements or the other light elements heavier than N (Qian \&
Wasserburg 2007). In this fact, the 'main $r$-process stars' CS 22892-052 and CS 31082-001 could have abundances that mainly reflect results of the main $r$-process nucleosynthesis of a few SNe. On the contrary, the 'weak $r$-process stars', i.e. HD 122563 and HD 88609, play an essential role in constraining the weak $r$-process, since they have the smallest contribution from the main $r$-process. Adopting the abundances of CS 22892-052 as the main $r$-process pattern, Zhang et al. (2010) found that, although the pattern of heavier neutron-capture elements is very similar to those of the main $r$-process pattern, the light-element abundance patterns of $r$-rich stars are close to those of weak $r$-process stars. In this study, based on the detailed abundance analysis of main $r$-process stars and weak $r$-process stars, we find that the abundances of light and iron group elements in main $r$ process stars do not come from main $r$-process events. We subtract the average abundances of main $r$-process stars from the average abundance of weak $r$-process stars, normalised to $\mathrm{Eu}$. We then subtract the obtained result from the average abundance of main $r$-process stars, normalised to Fe. Repeating the above processes, we can obtain the pure weak $r$ and main $r$-components, which had been scaled to the solar $r$-process abundances given by Arlandini et al. (1999) and the values of $\mathrm{Sr}-\mathrm{Nb}$ updated from Travaglio et al. (2004).

\section{RESULTS AND DISCUSSIONS}

\subsection{Fitted Results}

The observed abundances of nine stream stars are taken from Roederer et al. (2010a). We will explore the origin of the neutron-capture elements in the stars by comparing the observed abundances with predicted results. The $i$ th element abundance in a star can be calculated as

$$
N_{i}(Z)=\left(C_{r, m} N_{i, r, m}+C_{r, w} N_{i, r, w}\right) \times 10^{[\mathrm{Fe} / \mathrm{H}]},
$$

where $N_{i, r, m}$ and $N_{i, r, w}$ are the scaled abundances of the $i$ th element produced by the main $r$-process and weak $r$ process, respectively; $C_{r, m}$ and $C_{r, w}$ are the component coefficients corresponding to relative contributions from the main $r$-process and weak $r$-process to the elemental abundances, respectively. Based on Equation (1), using the observed data of stream stars (Roederer et al. 2010a), we can obtain the best-fit $C_{r, m}$ and $C_{r, w}$ by looking for the minimum $\chi^{2}$. The component coefficients and $\chi^{2}$ are listed in Table 1 . Figure 1 shows an example of our calculated best-fit results for the stream stars. The observed elemental abundances marked by filled circles are also shown to facilitate comparison. For most stars, there is good agreement between the predictions and the data for most elements starting with $\mathrm{O}$ to $\mathrm{Pb}$ in consideration of the observational errors. Roederer et al. (2010a) have suggested that the abundances of the stream stars do not contain the contribution of the $s$-process, except for the $s$-rich star CS 29513-032. Furthermore, because of the effect of the secondary-like nature of the major neutron source in massive stars, no weak $s$-contribution is expected 
Table 1. Component Coefficients and $\chi^{2}$ for Eight Stars of the Stellar Stream

\begin{tabular}{lcccc}
\hline \hline Star & {$[\mathrm{Fe} / \mathrm{H}]$} & $C_{r, m}$ & $C_{r, w}$ & $\chi^{2}$ \\
\hline BD+10 2495 & -2.31 & 1.81 & 3.77 & 0.99 \\
BD+29 2356 & -1.59 & 3.86 & 4.06 & 0.47 \\
BD+30 2611 & -1.50 & 4.49 & 2.83 & 0.61 \\
CD-36 1052 & -1.79 & 4.89 & 4.58 & 1.44 \\
HD 119516 & -2.26 & 3.09 & 3.24 & 1.52 \\
HD 128279 & -2.51 & 0.86 & 3.86 & 3.01 \\
HD 175305 & -1.73 & 3.42 & 4.41 & 0.82 \\
HD 237846 & -3.29 & 0.42 & 3.84 & 2.41 \\
\hline \hline
\end{tabular}

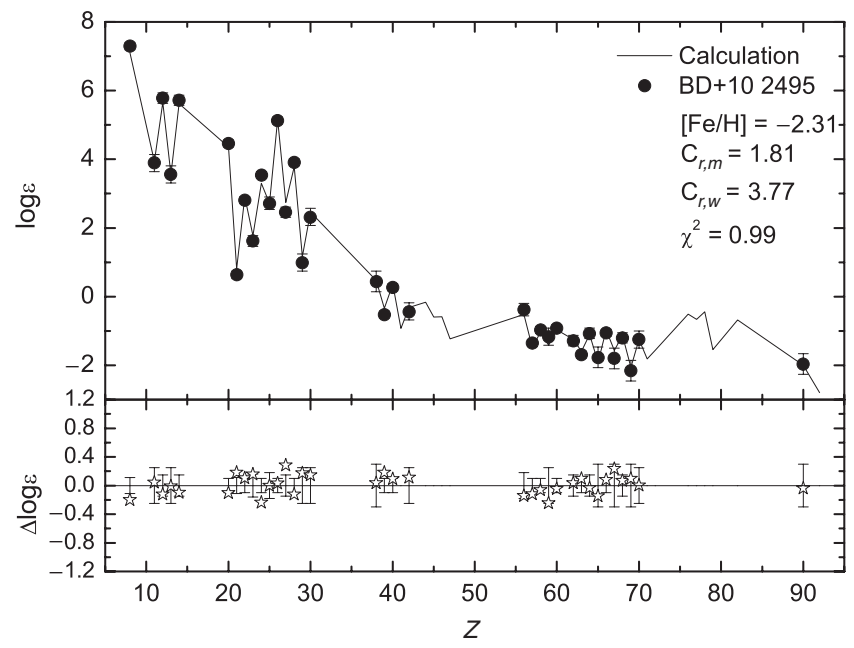

Figure 1. An example for the best-fit results of stream stars. In the top panel, the solid line presents our calculated result. The filled circles are observed elemental abundances. In the bottom panel, the open stars are individual relative offsets [ $\left.\Delta \log \varepsilon(X) \equiv \log \varepsilon(X)_{\text {cal }}-\log \varepsilon(X)_{\text {obs }}\right]$. The error bars are the observational errors.

in halo stars (Travaglio et al. 2004). As a test, adding the main $s$-process component and weak s-process component to Equation (1), we use four components to fit the abundances of metal-poor stars BD+29 $2356([\mathrm{Fe} / \mathrm{H}]=-1.59)$ and $\mathrm{BD}+302611([\mathrm{Fe} / \mathrm{H}]=-1.50)$. The main $s$-process pattern is taken from the result with $[\mathrm{Fe} / \mathrm{H}]=-2.0$ calculated by Busso et al. (2001) and the weak $s$-process pattern is taken from the result presented by Raiteri et al. (1993). The component coefficients deduced for BD+29 2356 are $C_{r, m}=3.85$, $C_{r, w}=4.14, C_{s, m}=0$, and $C_{s, w}=0$; for $\mathrm{BD}+302611, C_{r, m}$ $=4.49, C_{r, w}=2.82, C_{s, m}=0$, and $C_{s, w}=0.11$. We can see that the component coefficients of the main $s$-process and the weak $s$-process are smaller by one order of magnitude than those of the main $r$-and weak $r$-process. Our results are consistent with the suggestion presented by Roederer et al. (2010a) and Travaglio et al. (2004).

In the stream stars, CS 29513-032 shows clear enrichment by the $s$-process. Roederer et al. (2010a) have speculated that the $s$-process material observed in CS 29513-032 is accreted from another low-metallicity AGB star. If it is the case, the $s$-process abundance in the envelope of the stars could be expected to be lower than the abundance produced by the $s$-process in the AGB star because the material is mixed with the envelopes of the primary (former AGB star) and secondary stars. Roederer et al. (2010a) found that the observed abundances could not be fitted by the scaled solar system $r$-process pattern nor by the $s$-process pattern. We explore the origin of the neutron-capture elements in the $s$-rich stars by comparing the observed abundances with predicted $s$ - and $r$-process contribution. After considering the $s$-process contribution, the $i$ th element abundance in the envelope of the star can be calculated as follows:

$$
N_{i}(Z)=\left(C_{s} N_{i, s}+C_{r, m} N_{i, r, m}+C_{r, w} N_{i, r, w}\right) \times 10^{[\mathrm{Fe} / \mathrm{H}]},
$$

where $C_{s}$ is the component coefficients that correspond to the $s$-process and $N_{i, s}$ is the abundance produced by the $s$ process in the AGB star, which has been normalised to the $s$-process abundance of $\mathrm{Ba}$ in the solar system (Arlandini et al. 1999). The adopted abundance pattern $N_{i, s}$ in Equation (2) is taken from the main $s$-process abundance with $[\mathrm{Fe} / \mathrm{H}]=$ -3.0 for $1.5 \mathrm{M}_{\odot}$ given by Busso et al. (2001) (see their Figure 2 ). The fitted result (case A) is presented in the left panel of Figure 2. We can see that for most observed neutron-capture elements, their abundances can be fitted within the observational uncertainty. This means that the $s$-process elements in CS 29513-032 come from the dredged-up material in the former low-mass AGB star. The $r$-process coefficients and $\chi^{2}$ are $C_{r, m}=3.75, C_{r, w}=3.91$, and $\chi^{2}=1.3$. We can see that its $r$-process coefficients are close to those of other stream stars with similar metallicity. It is interesting to adopt the parametric model presented by Aoki et al. (2001) and developed by Zhang, Ma, and Zhou (2006) for s-rich stars to study the physical parameters which could reproduce the observed abundances. There are five parameters in the parametric model. They are the neutron exposure $\tau$, the overlap factor $r$, the component coefficient of the $s$-process $C_{s}$, and the component coefficients of the two $r$-processes $C_{r, w}, C_{r, m}$. Using the parametric approach, we can carry out $s$-process nucleosynthesis calculation to fit the abundance profile observed in the $s$-rich stars by looking for the minimum $\chi^{2}$ in the five-parameter space formed by $\tau, r, C_{s}, C_{r, w}$, and $C_{r, m}$. The right panel (case B) in Figure 2 shows our best-fit result. Because the parametric model contains more parameters, $\chi^{2}$ is smaller than that of case A.

In the top panel of Figure 3, we show individual relative offsets $(\Delta \log \varepsilon)$ for the sample stars with respect to the predictions. Typical observational uncertainties in $\log \varepsilon$ are $\sim 0.2-0.3$ dex (dotted lines). The root-mean-square (RMS) offsets of these elements in $\log \varepsilon$ shown in the bottom panel are mostly smaller than 0.30 dex for comparison with the calculated results. These values are consistent with zero, given by the combined uncertainties in stellar abundances and predicted abundances, which confirm the validity of the approach adopted in this work. From Figures 1-3 we can see that the agreement is obtained for both light elements, iron group elements and neutron-capture elements. 

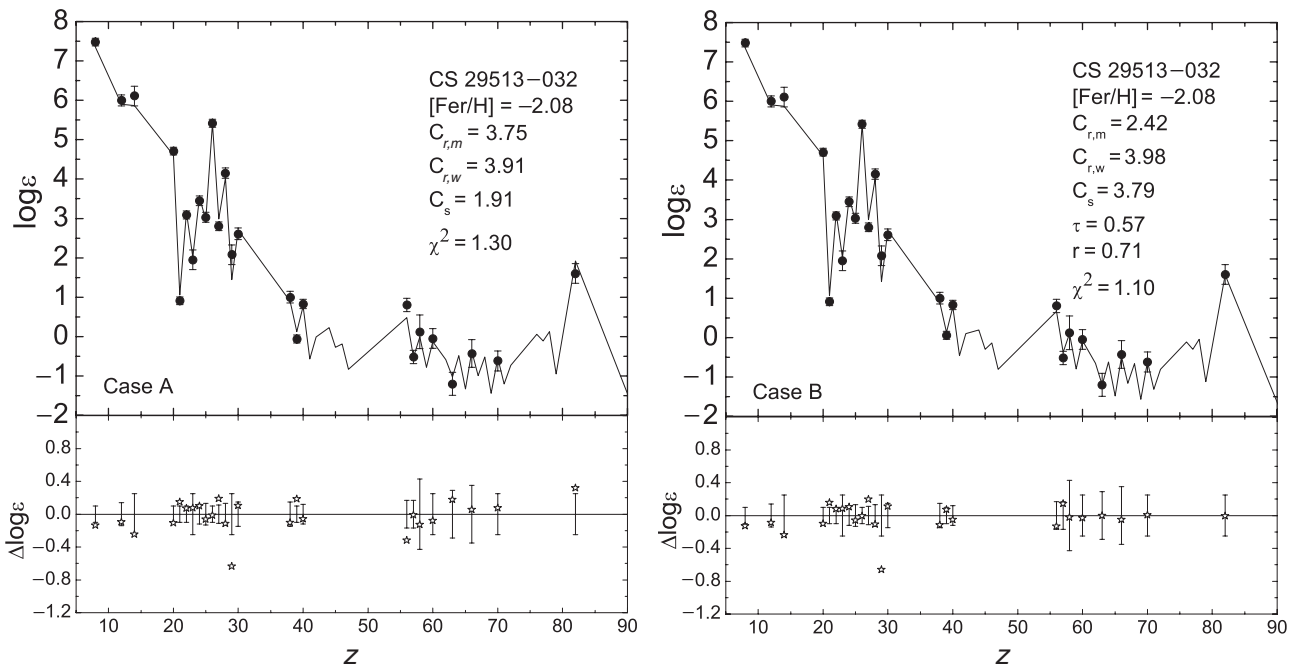

Figure 2. The best-fit results for CS 29513-032. The left panel shows our result by adopting the result of the AGB model with $[\mathrm{Fe} / \mathrm{H}]=-3$ given by Busso et al. (2001). The right panel shows our result by adopting the parametric approach. The symbols are the same as in Figure 1.

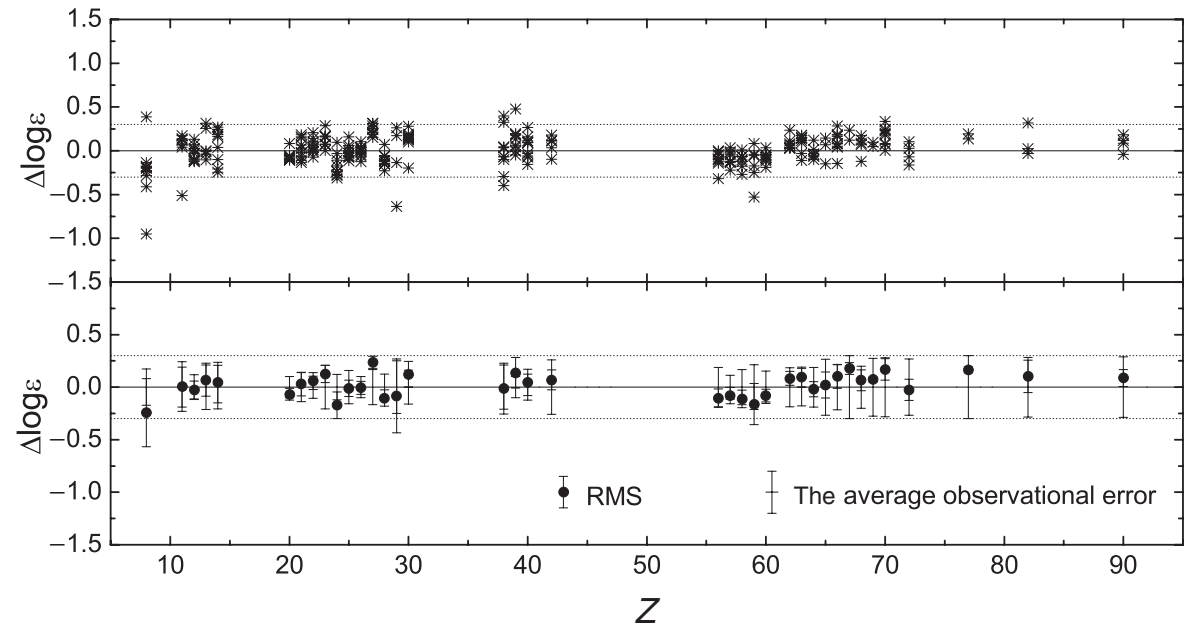

Figure 3. Top panel: individual relative offsets $\left[\Delta \log \varepsilon(X), \Delta \log \varepsilon(X) \equiv \log \varepsilon(X)_{\mathrm{cal}}-\log \varepsilon(X)_{\mathrm{obs}}\right]$ for the sample stars with respect to the predictions from the abundance model (stars). Typical observational uncertainties in $\log \varepsilon$ are $\sim 0.2-0.3$ dex (dotted lines). Bottom panel: the RMS offsets of these elements in $\log \varepsilon$. Filled circles are average stellar abundance offsets.

\subsection{The Two $r$-Process Component Coefficients}

The component coefficients as a function of metallicity, illustrated in Figure 4, contain some important information about the polluted history of the stellar stream. The trend of coefficients $C_{r, w}$ is almost constant for the stream stars, which is clearly different from that of $C_{r, m}$. The calculated results that $C_{r, w}$ is nearly constant suggest that the elements produced by the weak $r$-process have increased along with Fe over the polluted history of the stellar stream. This means that both the weak $r$-process elements and Fe are produced as primary elements from $\mathrm{SNe}$ II and their yields have nearly a constant mass fraction. Roederer et al. (2010a) have found that most of $\alpha$-elements and iron group elements have small or moderate dispersions, and show no evolution with metal- licity. This also means that both light elements and iron group elements are produced as primary elements from $\mathrm{SNe}$ II in which the weak $r$-process occurs, and their yields have nearly a constant mass fraction. On the other hand, they found that most of neutron-capture elements follow similarly increasing trends of [element $/ \mathrm{Fe}]$ ratios with increasing $[\mathrm{Fe} / \mathrm{H}]$. Obviously, the increased trend in $C_{r, m}$ as $[\mathrm{Fe} / \mathrm{H}]$ increases is a consequence of the gradual increase in the production of main $r$-process elements relative to iron. The rise means that the main $r$-process contribution may begin slightly later than the contribution from the weak $r$-process producing in conjunction with the Fe. This behavior supports the idea that the masses of progenitors for the main $r$-process are smaller than those of the weak $r$-process (or LEPP) (Travaglio et al. 1999, 


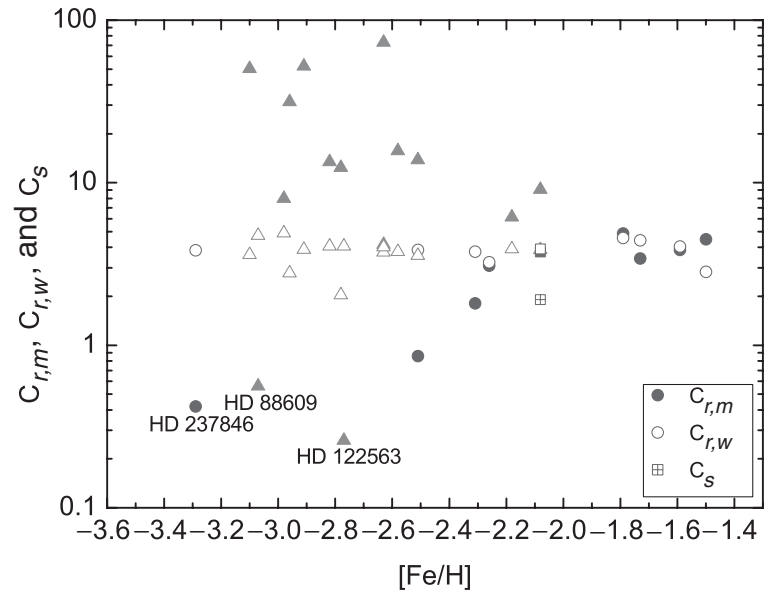

Figure 4. The component coefficients as a function of metallicity. Symbols: filled circles and open circles are the main $r$-process coefficients and the weak $r$-process coefficients of eight stream stars, respectively. Filled squares, open squares, and open squares with a cross are the component coefficients responsible for the maim $r$-process, weak $r$-process, and the main $s$-process in the $s$-rich star CS 29513-032, respectively. Filled triangles and open triangles are the main $r$-component coefficients and the weak $r$-component coefficients of 14 metal-poor halo stars studied in Zhang et al. (2010), respectively. Their abundances are observed by Westin et al. (2000), Cowan et al. (2002), Hill et al. (2002), Sneden et al. (2003), Honda et al. (2004, 2006, 2007), Barklem et al. (2005), Ivans et al. (2006), Christlieb et al. (2008), Hayek et al. (2009), Mashonkina et al. (2010), and Roederer et al. (2010b).

2004; Travaglio, Galli, \& Burkert 2001; Wanajo \& Ishimaru 2006; Qian \& Wasserburg 2007; Izutani et al. 2009). The main $r$-process coefficient $C_{r, m}$ is close to the weak $r$-process coefficient $C_{r, w}$ as the metallicity approaches $[\mathrm{Fe} / \mathrm{H}] \sim-2.2$.

For ease of comparison, the recalculated coefficients of 12 $r$-rich stars and two weak $r$-process stars (HD 122563 and HD 88609) studied in Zhang et al. (2010) are marked by triangles in Figure 4 . We find that their weak $r$-process component coefficients are close to those of the stream stars. However, for main $r$-process components, the difference between the stream stars with $[\mathrm{Fe} / \mathrm{H}]<-2.2$ and $r$-rich stars is obvious. As seen from Figure 4, the sample stars are separated into two branches clearly by $C_{r, m}$, i.e., $r$-rich stars having coefficient $C_{r, m}$ larger than 3.0 and 'low- $C_{r, m}$ ' stars with coefficient $C_{r, m}$ less than 3.0. All metal-poor stream stars $([\mathrm{Fe} / \mathrm{H}]<-2.2)$ and two weak $r$-process stars (HD 122563 and HD 88609) belong to 'low- $C_{r, m}$ ' stars.

\subsection{Discussion of the Abundance Decomposition}

As the abundances of the stream stars can be used as a probe of the origin of the neutron-capture elements in their progenitor system, it is important to determine the relative contributions from the individual neutron-capture processes to the abundances of heavy elements in the stars. It was possible to isolate the contributions corresponding to the weak $r$ - and main $r$-processes using our approach. Taking the values of $C_{r, m}$ and $C_{r, w}$ into Equation (1), the decomposed re- sults of sample stars can be derived. In Figures $5(\mathrm{a}-\mathrm{h})$, the component fractions $f_{i, r, m}\left(f_{i, r, m}=\frac{C_{r, m} N_{i, r, m}}{C_{r, m} N_{i, r, m}+C_{r, w} N_{i, r, w}}\right)$ and $f_{i, r, w}$ $\left(f_{i, r, w}=\frac{C_{r, w} N_{i, r, w}}{C_{r, m} N_{i, r, m}+C_{r, w} N_{i, r, w}}\right)$ as a function of $[\mathrm{Fe} / \mathrm{H}]$ are given by the filled circles (main $r$-process) and open circles (weak $r$ process), respectively. The abundances of $\mathrm{Sr}, \mathrm{Y}$, and $\mathrm{Zr}$ in the stream stars are a mixture of two $r$-process components. We can see that, for the sample star with the lowest metallicity, i.e. $[\mathrm{Fe} / \mathrm{H}]=-3.29$, the weak $r$-processes are dominantly responsible for abundances of the lighter neutron-capture elements $\mathrm{Sr}, \mathrm{Y}$, and $\mathrm{Zr}$. Although the contributions from weak $r$-processes are larger than those of the main $r$-process to the lighter neutron-capture elements of the stream stars, the contributions from the main $r$-process to lighter neutron-capture elements, such as $\mathrm{Sr}, \mathrm{Y}$, and $\mathrm{Zr}$, increase with increasing metallicity.

In order to study the origins of neutron-capture elements, the variation of the logarithmic ratio [element/Fe] with metallicity is particularly useful. The component ratios of the individual neutron-capture process, i.e., $[\mathrm{element} / \mathrm{Fe}]_{i}(i=r$, $w ; r, m)$ are derived and shown in Figure 6 . The filled circles and open circles respectively represent the main $r$-and weak $r$-component ratios. The trend of ratios [element $/ \mathrm{Fe}]_{r, w}$ is almost constant for the stream stars, which is clearly different from that of [element $/ \mathrm{Fe}]_{r, m}$. In particular, we focus on the ratios of $\mathrm{Sr}, \mathrm{Y}, \mathrm{Zr}$, which are partly produced by weak $r$ process nucleosynthesis. We can see that, for the sample star, the contributions from weak $r$-processes are higher than those of the main $r$-process for abundances of the lighter neutroncapture elements $\mathrm{Sr}$, Y, and $\mathrm{Zr}$. The calculated results that the $[\mathrm{Sr}, \mathrm{Y}, \mathrm{Zr} / \mathrm{Fe}]_{r, w}$ is nearly constant suggest that the elements produced by the weak $r$-process have increased along with Fe. This also means that both the weak $r$-process and Fe are produced as primary elements from SNe II and their yields have nearly a constant mass fraction. From Figure 6(a) we find that the ratios $[\mathrm{Sr} / \mathrm{Fe}]_{r, w}$ are nearly -0.3 . For the other lighter neutron-capture elements $\mathrm{Y}$ and $\mathrm{Zr}$, the similar results can be obtained. To investigate the contribution of the main $r$-process in [element/Fe] for heavier neutron-capture elements $\mathrm{Ba}, \mathrm{La}$, and $\mathrm{Eu}$, the variations of the logarithmic ratios $[\text { element } / \mathrm{Fe}]_{i}$ are shown in Figures 6(d-f). For the heavier neutron-capture elements, the figures show that the main $r$ process contribution is dominant. We find an increased trend in the $[\text { element } / \mathrm{Fe}]_{r, m}$ as $[\mathrm{Fe} / \mathrm{H}]$ increases. This would mean a consequence of the gradual increase in the production rate of the main $r$-process relative to iron due to the increased contribution of SNe II in which main $r$-process events occur. Our calculated results could be used as the constraints on the chemical characteristics of the progenitor system from which the stream stars originated.

Up to now, the metal-poor stars HD 122563 and HD 88609 are only two stars having extremely excesses of lighter neutron-capture elements and are called as 'weak $r$-process stars'. Their overall abundance pattern could represent the yields of the weak $r$-process. In order to investigate the robustness of the weak $r$-process pattern, it is very important 

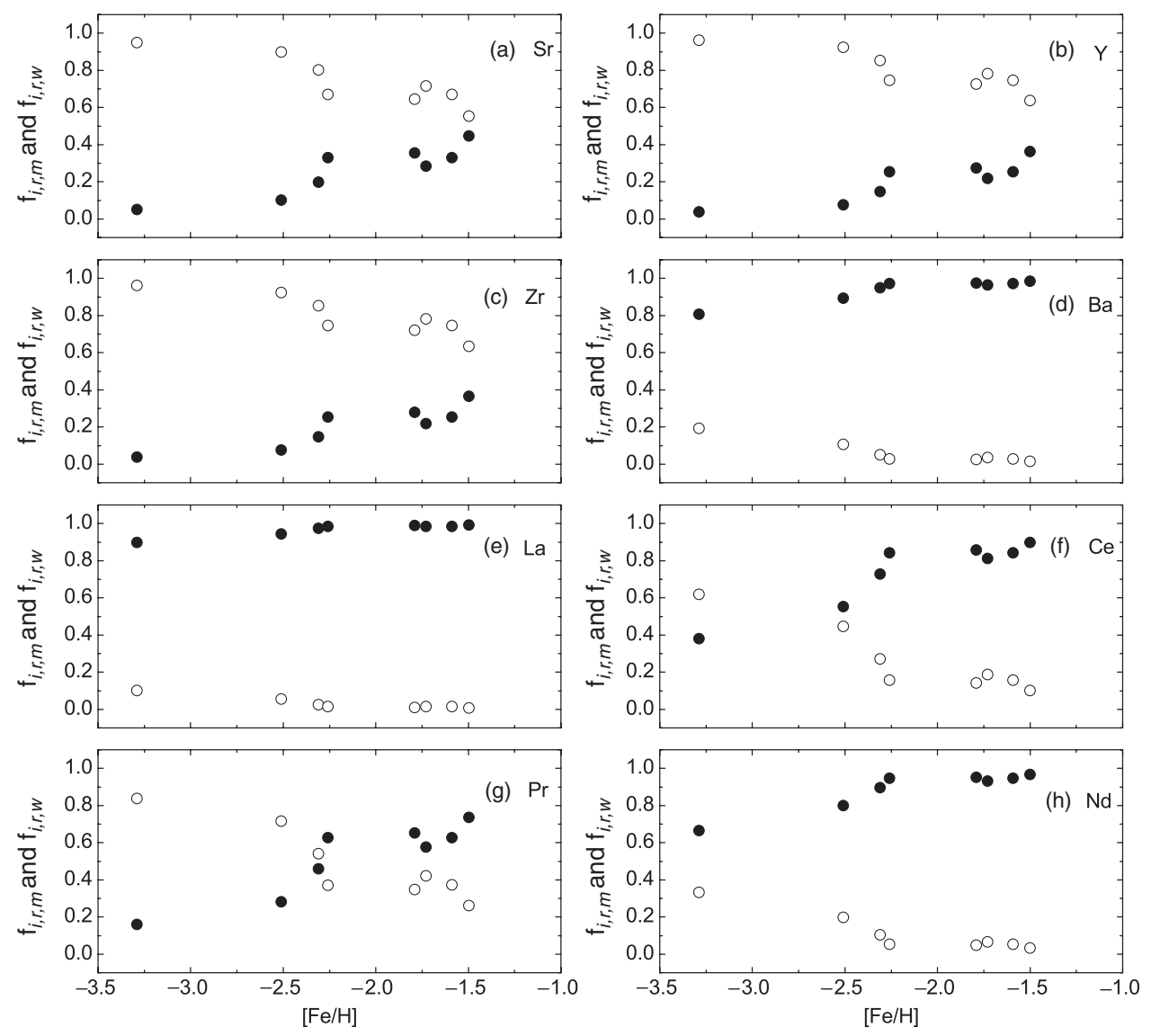

Figure 5. The component fractions of eight stream stars as a function of $[\mathrm{Fe} / \mathrm{H}]$. Symbols: filled circles and open circles are the fractions of the main $r$-component and the weak $r$-component, respectively.

to find the more weak $r$-process stars. The component coefficients can be used to select special stars. If one component coefficient is much larger than others, the abundances of this star may be dominantly produced by the corresponding process. Based on our results shown in Figure 4 and Table 1, we can find that the metal-poor stars, HD 237846 with $C_{r, m}=$ 0.42 and $C_{r, w}=3.84$, could be one weak $r$-process star. In order to test this finding, Figure 7 shows the abundance comparisons on the logarithmic scale between HD 237846 and HD 122563 as a function of the atomic number, which have been normalised to the Fe abundance of HD 122563. Our conclusion here is that, except element $\mathrm{O}$, the abundance patterns of HD 237846 and HD 122563 are quite similar. The metallicity of HD $237846([\mathrm{Fe} / \mathrm{H}]=-3.29)$ is even lower than that of HD 88609. This study reveals that HD 237846 is another weak $r$-process star.

\section{CONCLUSIONS}

In our Galaxy, nearly all chemical evolution and nucleosynthetic information is in the form of elemental abundances of stars with various metallicities. In this respect, the stellar abundances polluted by only a few processes, such as main $r$-process stars and weak $r$-process stars, are very sig- nificant, because their abundances can be directly compared with model prediction. However, such stars are very rare. The chemical abundances of the metal-poor stars in the stellar stream are excellent information for setting constraints on models of $r$-processes. Our results can be summarised as follows.

1. The observed abundances of the heavy elements in the stream stars cannot be well fitted by the abundances of the main $r$-process (Roederer et al. 2010a). After adding the contribution of the weak $r$-process and its accompaniment, the abundances of most metal-poor stream stars, including light elements, iron group elements, and neutron-capture elements, can be fitted. For lighter neutron-capture elements $\mathrm{Sr}, \mathrm{Y}$, and $\mathrm{Zr}$, the contributions from the weak $r$-process are larger than those of the main $r$-process.

2. The weak $r$-process coefficients are almost constant for the sample stars, including $r$-rich stars. This means that the elements produced by the weak $r$-process have increased along with $\mathrm{Fe}$ over the polluted history of the stellar stream. Both the weak $r$-process and $\mathrm{Fe}$ are produced as primary elements from SNe II and their yields have nearly a constant mass fraction. 

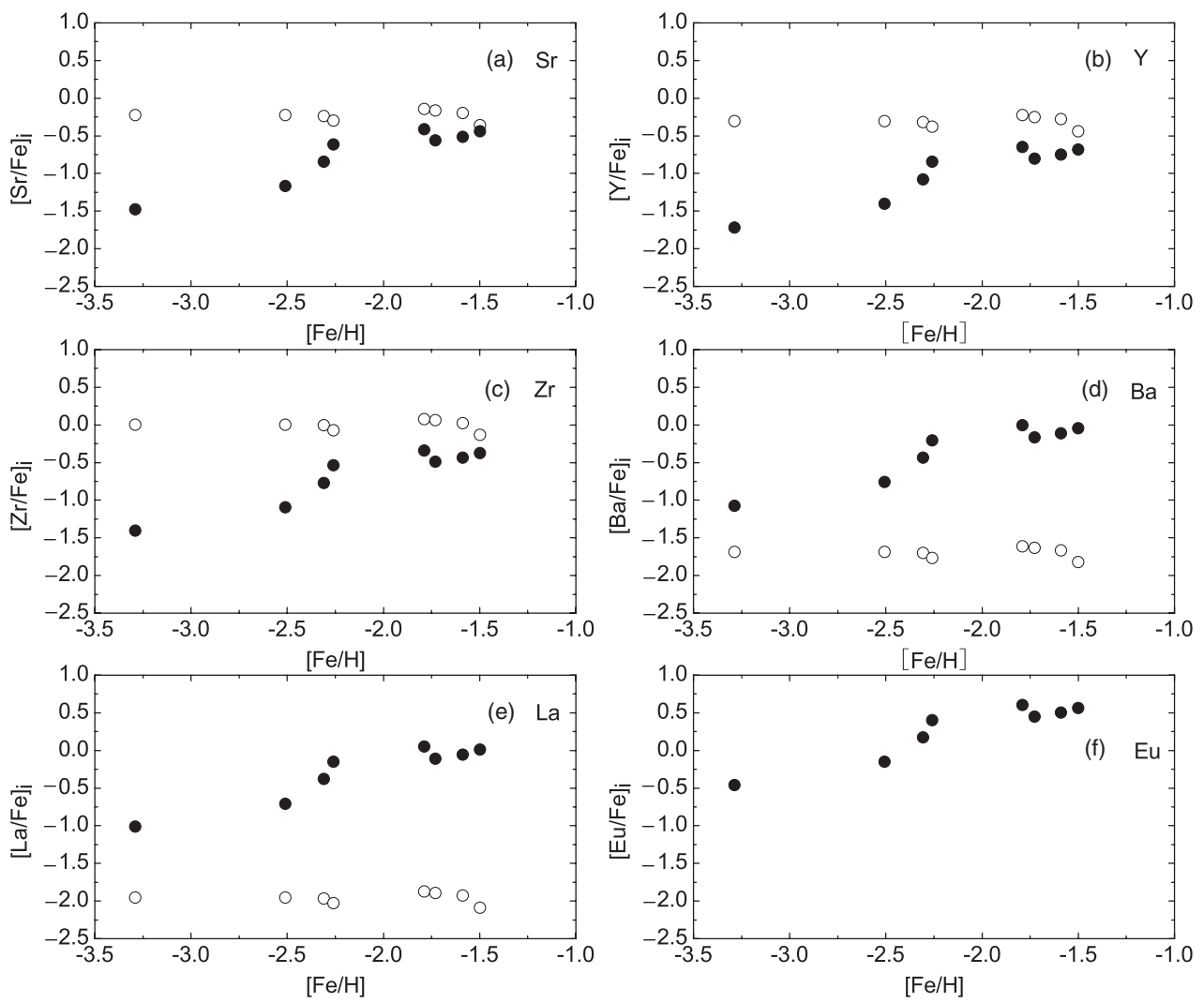

Figure 6. The logarithmic component ratios vary with metallicity for eight stream stars. Symbols: filled circles and open circles are the main $r$-component ratios and the weak $r$-component ratios, respectively.

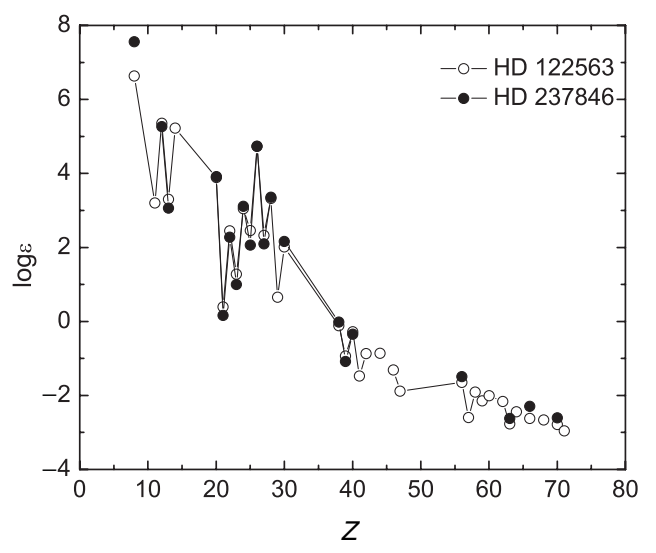

Figure 7. The abundance comparisons on the logarithmic scale between HD 237846 and HD 122563 as a function of the atomic number. Symbols: the open circles and filled circles present the observed abundances in HD 122563 and HD 237846, respectively.

3. The increased trend in the $[\mathrm{element} / \mathrm{Fe}]_{r, m}$ as $[\mathrm{Fe} / \mathrm{H}]$ increases means the gradual increase in the production rate of the main $r$-process relative to iron. This behavior is due to the increased contribution of SNe II in which main $r$-process events occur and would imply that the masses of progenitors for the main $r$-process are smaller than those of the weak $r$-process.
4. The difference between the stream stars and $r$-rich stars is obvious. The $r$-rich stars and the stream stars are separated into two branches clearly, with a systematic different in the main $r$-process component $C_{r, m}$ at a given metallicity. All metal-poor stream stars $([\mathrm{Fe} / \mathrm{H}]$ $<-2.2$ ) belong to 'low- $C_{r, m}$ ' stars. Moreover, the weak $r$-process stars HD 122563 and HD 88609 also belong to 'low- $C_{r, m}$ ' stars.

5. Although a large number of metal-poor stars have been studied over the past few decades, only two weak $r$ process stars, HD $122563([\mathrm{Fe} / \mathrm{H}]=-2.77)$ and $\mathrm{HD}$ $88609([\mathrm{Fe} / \mathrm{H}]=-3.07)$, have been found. Using the component coefficients, chemical-specific stars can be selected. We find that the metal-poor star HD 237846 is another weak $r$-process star. The metallicity of HD 237846 is $[\mathrm{Fe} / \mathrm{H}]=-3.29$, which means that the abundance pattern produced by the weak $r$-process is stable from star to star for a wider range of metallicities. This result would give a stronger constraint on the models of the weak $r$-process in the very early Galaxy.

6. The calculated results imply that the relative contribution from the individual neutron-capture process to the heavy element abundances is not usually in the solar proportion, for most stream stars. The contributions of the weak $r$-process are larger than those of the main $r$-processes to lighter neutron-capture elements $\mathrm{Sr}, \mathrm{Y}$, 
and $\mathrm{Zr}$. The main $r$-process coefficient $C_{r, m}$ close to the weak $r$-process coefficient $C_{r, w}$ as the metallicity approaches $[\mathrm{Fe} / \mathrm{H}] \sim-2.2$, which implies that both the weak $r$-process and the main $r$-process component fractions reach their solar system values as the metallicity approaches $[\mathrm{Fe} / \mathrm{H}] \sim-2.2$.

7. CS $29513-032$ is the only $s$-rich star in the stellar stream. Considering the contribution of the $s$-process, its abundances can be fitted. The $s$-process elements in CS 29513-032 are a result of pollution from the dredged-up material in the former low-mass AGB star (e.g. $1.5 \mathrm{M}_{\odot}$ ). Based on the analysis of angular momentum components, Roederer et al. (2010a) suggested the $s$-rich star CS 29513-032 is a member of the stream stars. We find that its $r$-process coefficients are close to other stream stars with similar metallicity, which means that the initial abundance (i.e. before polluted by the AGB star) of CS 29513-032 is similar to the abundances of the other stream stars. This implies that the astrophysical origin of CS 29513-032 and other stream stars is similar. Our result supports the suggestion by Roederer et al. (2010a). CS 29513-032 should belong to a wide binary system which forms in a molecular cloud that has been polluted by both the weak and main $r$-process material.

Clearly, it is important for future studies to determine the relation between metal-poor halo stars and metal-poor stellar stream stars. More theoretical and observational studies of stream stars will reveal the characteristics of the $r$-process at low metallicity and the history of the enrichment of neutroncapture elements in the early Galaxy.

\section{ACKNOWLEDGMENTS}

We are grateful to the referee for very valuable comments and suggestions that improved this paper greatly. This work has been supported by the National Natural Science Foundation of China under grants U1231119, 11273011, 10847119, 10778616, 10973006, and 11003002 , the Natural Science Foundation of Hebei Provincial Education Department under grant 2008127, the Science Foundation of Hebei Normal University under grants L2007B07 and L2009Z04, the Natural Science Foundation of Hebei Province under grants A2009000251 and A2011205102, and the Program for Excellent Innovative Talents in the University of Hebei Province under grant CPRC034.

\section{REFERENCES}

Aoki, W., et al. 2001, ApJ, 561, 346

Arlandini, C., Käppeler, F., Wisshak, K., Gallino, R., Lugaro, M., Busso, M., \& Straniero, O. 1999, ApJ, 525, 886

Barklem, P. S., et al. 2005, A\&A, 439, 129

Burbidge, E. M., Burbidge, G. R., Fowler, W. A., \& Hoyle, F. 1957, RvMP, 29, 547

Busso, M., Gallino, R., Lambert, D. L., Travaglio, C., \& Smith, V. V. 2001, ApJ, 557, 802
Busso, M., Gallino, R., \& Wasserburg, G. J. 1999, ARA\&A, 37, 239

Christlieb, N., Schörck, T., Frebel, A., Beers, T. C., Wisotzki, L., \& Reimers, D. 2008, A\&A, 484, 721

Cowan, J. J. \& Sneden, C. 2006, Natur, 440, 1151C

Cowan, J. J., Thielemann, F.-K., \& Truran, J. W. 1991, PhR, 208, 267

Cowan, J. J., Pfeiffer, B., Kratz, K.-L., Thielemann, F.-K., Sneden, C., Burles, S., Tytler, D., \& Beers, T. C. 1999, ApJ, 521,194

Cowan, J. J., et al. 2002, ApJ, 572, 861

Cowan, J. J., et al. 2005, ApJ, 627, 238

Eggen, O. J., Lynden-Bell, D., \& Sandage, A. R. 1962, ApJ, 136, 748

Hayek, W., et al. 2009, A\&A, 504, 511

Helmi, A., White, S. D. M., de Zeeuw, P. T., \& Zhao, H. 1999, Natur, 402, 53

Hill, V., et al. 2002, A\&A, 387, 560

Honda, S., Aoki, W., Ishimaru, Y., \& Wanajo, S. 2007, ApJ, 666, 1189

Honda, S., Aoki, W., Ishimaru, Y., Wanajo, S., \& Ryan, S. G. 2006, ApJ, 643, 1180

Honda, S., Aoki, W., Kajino, T., Ando, H., Beers, T. C., Izumiura, H., Sadakane, K., \& Takada-Hidai, M. 2004, ApJ, 607, 474

Ishimaru, Y., Wanajo, S., Aoki, W., Ryan, S. G., \& Prantzos, N. 2005, Nucl. Phys. A, 758, 603

Ivans, I., Simmerer, J., Sneden, C., Lawler, J. E., Cowan, J. J., Gallino, R., \& Bisterzo, S. 2006, ApJ, 645, 613

Izutani, N., Umeda, H., \& Tominaga, N. 2009, ApJ, 692, 1517

Kepley, A. A., et al. 2007, AJ, 134, 1579

Mashonkina, L., Christlieb, N., Barklem, P. S., Hill, V., Beers, T. C., \& Velichko, A. 2010, A\&A, 516, 46

Montes, F., et al. 2007, ApJ, 671, 1685

Qian Y.-Z. \& Wasserburg, G. J. 2007, PhR, 442, 237

Raiteri, C. M., Gallino, R., Busso, M., Neuberger, D., \& Kappeler, F. 1993, ApJ, 419, 207

Roederer, I. U., Sneden, C., Lawler, J. E., \& Cowan, J. J. 2010b, ApJ, 714, L123

Roederer, I. U., Sneden, C., Thompson, I. B., Preston, G. W., \& Shectman, S. A. 2010a, ApJ, 711, 573

Searle, L. \& Zinn, R. 1978, ApJ, 225, 357

Serminato, A., Gallino, R., Travaglio, C., Bisterzo, S., \& Straniero, O. 2009, PASA, 26, 153

Sneden, C., Cowan, J. J., \& Gallino, R. 2008, ARA\&A, 46, 241

Sneden, C., Cowan, J. J., Ivans, I. I., Fuller, G. M., Burles, S., Beers, T. C., \& Lawer, J. E. 2000, ApJ, 533, L139

Sneden, C., et al. 2003, ApJ, 591, 936

Truran, J. W., Cowan, J. J., Pilachowski, C. A., \& Sneden, C. 2002, PASP, 114, 1293

Travaglio, C., Galli, D., \& Burkert, A. 2001, ApJ, 547, 217

Travaglio, C., Gallino, R., Arnone, E., Cowan, J., Jordan, F., \& Sneden, C. 2004, ApJ, 601, 864

Travaglio, C., Galli, D., Gallino, R., Busso, M., Ferrini, F., \& Straniero, O. 1999, ApJ, 521, 691

Wanajo, S. \& Ishimaru, Y. 2006, Nucl. Phys. A, 777, 676

Westin, J., Sneden, C., Gustafsson, B., \& Cowan, J. J. 2000, ApJ, 530,783

Zhang, J., Cui, W., \& Zhang, B. 2010, MNRAS, 409, 1068

Zhang, B., Ma, K., \& Zhou, G. 2006, ApJ, 642, 1075 